\title{
Competencias de Docentes y Acreditación en la Universidad Nacional José Faustino Sánchez Carrión, 2014
}

\author{
Competencies of Teachers and the National Accreditation José Faustino Sánchez Carrión \\ University, 2014 \\ Carlos Bernardino Ruiz Huaraz ${ }^{1}$, Zoila Gregoria Rueda Bazalar ${ }^{2}$, Edgardo Octavio Carreño Cisneros ${ }^{3}$, Irina \\ Patricia Calvo Rivera ${ }^{1}$.
}

\section{RESUMEN}

Objetivo: Determinar la relación que existe entre las competencias docentes y el proceso de acreditación de las escuelas académico profesionales de la Universidad Nacional José Faustino Sánchez Carrión. Métodos: La población de estudio fueron 548 docentes de las diferentes escuelas académicas profesionales seleccionados mediante el muestreo probabilístico, se utilizó el instrumento de medición de actitudes de escala de Likert. Considerándose como dimensiones: Pensamiento estratégico, Compromiso, liderazgo. La confiabilidad de los instrumentos fue validado mediante el coeficiente alfa de Cronbach $(0,945$ y 0,960). La Prueba de Rho de Spearman. Resultados: Los resultados muestran que el mayor porcentaje (45\%) de docentes están muy de acuerdo en la competencia docente en las escuelas académicas, en cuanto al proceso de acreditación en las escuelas académico profesionales reporta un porcentaje mayor de $46,8 \%$ con una opinión algo de acuerdo. Conclusión: Los resultados obtenidos demuestran que existe una correlación positiva significativa moderada entre las competencias docentes y los procesos de acreditación (Rho=0,592; $\mathrm{p}=0,00<0,05)$.

Palabras clave: Autoevaluación, compromiso, liderazgo, pensamiento estratégico.

\section{ABSTRACT}

Objective: To determine the relationship between teaching skills and the process of accreditation of professional academic schools of the National University José Faustino Sánchez Carrion. Methods: The study population consisted of 548 teachers from different academic and professional schools selected by probability sampling, the measuring attitudes Likert scale was used. Considered as dimensions: strategic thinking, commitment, leadership. The reliability of the instrument was validated using Cronbach's alpha coefficient (0.945 and 0.960). Rho the Spearman Test. Results: The results show that the highest percentage (45\%) Teachers are strongly agreed teaching competence in academic schools, regarding the accreditation process in academic professional schools reported a higher percentage of $46.8 \%$ with an opinion somewhat agree. Conclusion: The results demonstrate a significant moderate positive correlation between educational competencies and accreditation processes $($ Rho $=0.592 ; p=0.00<0.05)$.

Keywords: Self-Assessment, commitment, leadership, strategic thinking.

\footnotetext{
${ }^{1}$ Facultad de Ciencias Económicas, Contables y financieras. Universidad Nacional José Faustino Sánchez Carrión. Huacho, Perú

${ }^{2}$ Facultad de Ciencias Sociales. Universidad Nacional José Faustino Sánchez Carrión. Huacho, Perú

${ }^{3}$ Facultad de Ingeniería Química y Metalúrgica. Universidad Nacional José Faustino Sánchez Carrión. Huacho, Perú
} 


\section{INTRODUCCIÓN}

Los cambios que se vienen produciendo en la sociedad del conocimiento requiere de una educación que genere el desarrollo social, económico y cultural y a la vez la clave para lograr el pleno ejercicio de la democracia y la equidad social (Del Basto, 2004). Su labor formativa no se agota en el desarrollo de competencias para la investigación científica y sus aplicaciones prácticas, abarca los espacios de aprendizaje, socialización e interacción permanente. La misión de la universidad, es tener un modelo de hombre y mujer que articule su formación profesional con su sensibilidad estética, su dimensión psicoafectiva y su conciencia ética, para ello es necesario que se cuente con docentes universitarios competentes y que garanticen la misión de la universidad.

Las universidades acreditadas deben responder a una serie de factores que determinen su calidad, en el contexto de la globalización, de la internacionalización y debe asumir el desafío de la modernidad e integrarse exitosamente a esta sociedad del saber. Sin embargo en nuestra realidad universitaria, sobre todo en las últimas décadas se evidencia la proliferación y diversificación de instituciones de educación superior, muchas de ellas se han creado sin cumplir los requisitos de calidad, para su respectiva acreditación de los programas académicos que ofrecen. La acreditación garantiza a la sociedad que el quehacer de las universidades responde a las necesidades de la sociedad con calidad, por lo tanto la acreditación es un medio para el logro de la calidad y el mejoramiento institucional.

En ese contexto el gobierno peruano no se encuentra ajeno a esta realidad, es así que en un escenario de globalización de competencia ha emitido el año 2006 la Ley No 28740 del Sistema Nacional de Evaluación, Acreditación y Certificación de la Calidad Educativa a fin de normar los procesos de evaluación, acreditación y certificación de la calidad educativa, y es a través de CONEAU el órgano operador encargado de definir los criterios, indicadores y estándares de medición para garantizar en las universidades públicas y privadas los niveles aceptables de calidad así como alentar la aplicación de las medidas requeridas para su mejoramiento.
Dentro de ese marco de calidad para la acreditación, la evaluación del recurso humano en una institución superior universitaria es de vital importancia, puesto que señala los objetivos alcanzados y vislumbra el camino a seguir para continuar perfeccionando todos los elementos que lo componen en pro de la mejora continua y el alcance de los estándares de calidad. De ahora en adelante las universidades tienen que aplicar el modelo de calidad para la acreditación de las carreras profesionales universitarias, con lo que se estaría asegurando la calidad de la formación profesional.

Según Vásquez, García y Cano (2013), en su estudio ¿Las competencias de los coordinadores impacta la acreditación de los programas de posgrado? identificaron las competencias de los coordinadores de programas de posgrado, entre las que destacaron resolución de problemas, orientación a resultados, trabajo en equipo. Por otro lado Marcano (2006), manifiesta que las prácticas actuales exigen cada vez más docentes con un alto nivel de desarrollo profesional, capaces de enfrentar los nuevos retos y transformaciones de la sociedad. Las competencias representan una combinación dinámica de conocimiento, comprensión, capacidades y habilidades. Las competencias se forman en varias unidades del curso y son evaluadas en diferentes etapas. Pueden estar divididas en competencias relacionadas con un área de conocimiento (específicas de un campo de estudio) y competencias genéricas (comunes para diferentes cursos) Tuning América Latina (2007).

\section{Las hipótesis en estudio fueron:}

Ha: Existe relación directa entre las competencias docentes y el proceso de acreditación de las escuelas académico profesionales de la Universidad Nacional José Faustino Sánchez Carrión.

Ho: No Existe relación directa entre las competencias docentes y el proceso de acreditación de las escuelas académico profesionales de la Universidad Nacional José Faustino Sánchez Carrión.

Por otro lado, esta experiencia forma parte del proceso de acreditación de nuestra universidad y que permite compartirla con otras universidades para una mejora continua, y se 
constituya en orientación para otras experiencias educativas en el nivel universitario, de esta manera se justificaría la investigación realizada. Teniendo como objetivo determinar la relación que existe entre las competencias docentes y el proceso de acreditación de las escuelas académico profesionales de la Universidad Nacional José Faustino Sánchez Carrión.

\section{MATERIAL Y MÉTODOS}

Para la ejecución de la investigación se utilizó el tipo de estudio correlacional transversal. El área de estudio considerado fue la Universidad Nacional José Faustino Sánchez Carrión. La población de estudio fueron 548 docentes, seleccionadas mediante muestreo por conveniencia con un tamaño muestral de 111 distribuidos proporcionalmente al tamaño de la población. El instrumento de medición de actitudes estuvo basado en la escala tipo Likert. El procesamiento de dato una vez de haber terminado de recolectar los datos utilizando el Software Estadístico Statistical Packege Social Sciencies (SPSS) versión 22 y el software de hoja de cálculo Microsoft Office Excel 2010.

Para el procesamiento del cuestionario se utilizó la escala Likert, cuya puntuación por ítems corresponde a las respuestas dadas por los docentes de las diversas escuelas académico profesionales de la universidad, asignándose el siguiente puntaje, Nunca $=1$; $\mathrm{P}$ oc a s vece $\mathrm{s}=2 ;$ a $\mathrm{veces}=3$; frecuentemente $=4 ;$ siempre $=5$

Para determinar la confiabilidad del instrumento se calculó el Alpha de Cronbach. El instrumento mostró 40 ítems, distribuidos en 21 ítems para la variable independiente y de 20 ítems para la variable dependiente, considerando a los 111 individuos (docentes) encuestados: Para el instrumento de la variable independiente y dependiente se obtuvo un coeficiente de confiabilidad Alpha de Cronbach de 0,945 y 0,960 respectivamente, lo que puede interpretarse como un instrumento de alta confiabilidad.

Los datos se recodificaron para determinar algunas variables para las pruebas de independencia con Chi cuadrada (x2). Para la realización de la prueba y con la finalidad de cumplir con el mínimo de 5 observaciones para las celdas, se agruparon las respuestas de Muy en desacuerdo, algo en desacuerdo, ni de acuerdo ni en desacuerdo, algo de acuerdo y muy de acuerdo. Se empleó la prueba de Chi cuadrado y la Prueba de Rho de Spearman.

\section{RESULTADOS}

Con el fin de comprobar la hipótesis nula se aplicó la prueba chi cuadrado dando por resultado que se rechaza la hipótesis general nula con un $\alpha=0,000$ se rechazó, aceptando que existe relación directa entre las competencias docentes y el proceso de acreditación de las escuelas académico profesionales de la Universidad Nacional José Faustino Sánchez Carrión.

Asimismo se acepta que existe relación directa entre las variables pensamiento estratégico y el proceso de acreditación. Y que existe relación directa entre el liderazgo y el proceso de acreditación hay relación directa entre el pensamiento estratégico y el proceso de acreditación, de las Escuelas Académico Profesionales de la Universidad Nacional José Faustino Sánchez Carrión.

Tabla 1. Valores obtenidos de la prueba de Chi Cuadrada $\left(X^{2}\right)$ evaluada

\begin{tabular}{lcccc}
\hline $\begin{array}{l}\text { Variables e } \\
\text { Indicadores }\end{array}$ & $\begin{array}{c}\text { Valor de } \\
\text { Chi Cuadrada }\end{array}$ & $\begin{array}{c}\text { Grados de } \\
\text { Libertad }\end{array}$ & $\begin{array}{c}\text { Nivel de significancia } \\
\text { Observado (Valor P) }\end{array}$ & Ho \\
\hline Docentes & & 12 & 0,00 & Se rechaza \\
Competencias docentes & 87,682 & 12 & 0,00 & Se rechaza \\
Pensamiento estratégico & 80,867 & 12 & Se rechaza \\
Compromiso & 40,019 & 16 & 0,00 & Se rechaza \\
Liderazgo & 95,012 & & & \\
\hline
\end{tabular}


En la Tabla 1, Se muestra que la hipótesis nula se rechaza al nivel de error del $5 \%$; es decir que existe independencia significativa entre cada una de las dimensiones de las variables.

\section{Docentes}

En relación a las actitudes de los docentes con respecto al pensamiento estratégico para los procesos de acreditación el $45 \%$ está muy de acuerdo; así mismo se observó que el 53,2\% de los docentes manifiesta estar muy de acuerdo en el compromiso, mientras que el $0,0 \%$ cree estar muy en desacuerdo, por otro lado el $46,8 \%$ de los docentes cree estar algo de acuerdo en el liderazgo, mientras que el $2,7 \%$ cree estar muy en desacuerdo.

\section{Consolidado}

Al relacionar las variables competencias docentes y el proceso de acreditación para escuelas académico profesionales de la Universidad Nacional José Faustino Sánchez Carrión, los docentes opinaron que el 45,0\% está muy de acuerdo en que son importantes las competencias, el 42,3\% manifiestan estar algo de acuerdo el 8,1\% manifiestan ni de acuerdo ni en desacuerdo, mientras que el 4,5\% manifiestan estar algo en desacuerdo; así mismo con respecto a la variable procesos de acreditación el $36 \%$ de los docentes manifiestan estar muy de acuerdo, el $46,8 \%$ algo de acuerdo, con un $12,6 \%$ ni de acuerdo ni en desacuerdo, el 1,8\% muy en desacuerdo.

Por lo que el $87,3 \%$ de los docentes están de acuerdo en las competencias docentes; así mismo el $82,8 \%$ están de acuerdo en los procesos de acreditación.

En cuanto a la prueba de hipótesis general para indicar las relaciones de sus variables se ejecutó mediante el valor $\alpha=5 \%$ para lo cual requirió de las siguientes interpretaciones: $\mathrm{Si}$ valor $p \geq 0,05$ se acepta hipótesis nula (Ho); si $p$ $<0,05$ se acepta hipótesis de investigación o hipótesis alternativa ( $\mathrm{Ha})$. El estadístico de prueba fue la correlación Rho de Spearman, dado que este estadístico es apropiado para ver relaciones entre variables, obteniéndose los siguientes resultados:

Tabla 2. Correlaciones de variables

\begin{tabular}{|c|c|c|c|}
\hline & & $\begin{array}{l}\text { Variable Independiente } \\
\text { Competencias docente }\end{array}$ & $\begin{array}{l}\text { Variable Dependiente } \\
\text { Proceso de acreditación }\end{array}$ \\
\hline Variable & Rho de Spearman & 1 & $0,592^{\star *}$ \\
\hline Independiente & Sig. (bilateral) & &, 000 \\
\hline $\begin{array}{l}\text { Competencias } \\
\text { docente }\end{array}$ & $\mathrm{N}$ & 111 & 111 \\
\hline \multirow{5}{*}{$\begin{array}{l}\text { Variable } \\
\text { Dependiente } \\
\text { Procesos } \\
\text { acreditación }\end{array}$} & Coeficiente de & & \\
\hline & correlación Rho de & $0,592^{* *}$ & 1 \\
\hline & Spearman & & \\
\hline & Sig. (bilateral) &, 000 & \\
\hline & $\mathrm{N}$ & 111 & 111 \\
\hline
\end{tabular}

**. La correlación es significativa en el nivel 0,01 (2 colas).

El Valor de $p=0,000$ Rho de Sperman $=0,592$

Con una probabilidad de error del $0,000 \%$ menor que 0,05 existe una correlación positiva moderada y significativa entre las variables competencias docentes y procesos de acreditación.

\section{DISCUSIÓN}

La opinión de los docentes respecto a las competencias docentes y procesos de acreditación de las carreras profesionales analizadas pone en evidencia los siguientes aspectos.

Las competencias docentes influyen directamente en los procesos de acreditación, como señala Corvalán (2010), que es necesario establecer una estrategia para la formación docente con una selección competente para logra la calidad y su acreditación

Cuando nuestra universidad realizó su primera autoevaluación (2011) de las carreras profesionales, salió desaprobada en los estándares de las dimensiones: Gestión de la carrera de la formación profesional y de los servicios de apoyo para la formación profesional.

Una vez obtenidos y analizados los resultados de las investigaciones, nos permite aceptar la hipótesis general, es decir existe relación directa entre las competencias docentes y los procesos de acreditación de las Escuelas Académico 
Profesionales de la Universidad Nacional José Faustino Sánchez Carrión.

Con relación a la hipótesis especifica uno, se desprende del análisis de correlación entre las variables, planeamiento estratégico y procesos de acreditación de las Escuelas Académico Profesionales de la Universidad Nacional José Faustino Sánchez Carrión hay una relación, ya que al aplicarse la prueba de hipótesis se obtuvo el resultado de $\alpha=0,00$ que es menor a $\alpha(0,05)$.

Con respecto a la segunda hipótesis se afirma que existe una probabilidad en que el compromiso influye directamente en los procesos de acreditación de las Escuelas Académico Profesionales de la Universidad Nacional José Faustino Sánchez Carrión, según los datos de significancia $(0,000)$ obtenidos

Con respecto a la tercera hipótesis se acepta porque existe un liderazgo que influye positivamente en los procesos de acreditación de las Escuelas Académico Profesionales de la Universidad Nacional José Faustino Sánchez Carrión.

\section{REFERENCIAS BIBLIOGRÁFICAS}

Corvalán, O. (2010). La profesionalización docente, condición para la carrera docente. Revista Electrónica desarrollo de competencias (REDEC), 6(2), 124-151
Del Basto, L. (2004). Una Reflexión en torno a la universidad y su acreditación. En revista electrónica de la red de investigación educativa, ieRed, Vol. 1(1), 1-12

Marcano, L. (2006). "Modelo de Evaluación del Desempeño Profesional Docente". Tesis para optar el Grado Científico de Doctor en Ciencias Pedagógicas, Santa Clara: Universidad Pedagógica Félix Varela.

Vásquez, A., García, T. \& Cano, M. (2013). ¿Las competencias de los coordinadores impacta la acreditación de los programas de posgrado? Revista International Administración \& Finanzas, 6(4), 89-103.

Tuning América Latina (2007). Reflexiones y perspectivas de la Educación Superior en América Latina. Informe. España: Publicaciones de la Universidad de Deusto.

Correo electrónico: cruoz@unjfsc.edu.pe

Revisión de Pares:

Recibido: 21-05-2015

Aceptado: 21-12-2015 